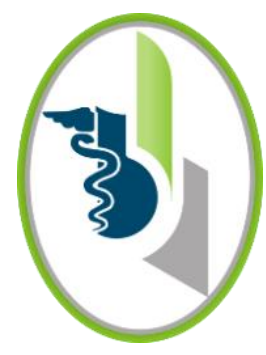

ACCESO abierto

Para citaciones: Manrique, E., Lozada, I., García, M. (2020). Frecuencia de patologías orgánicas derivadas del consumo de sustancias psicoactivas en pacientes de una Unidad de Cuidados Intensivos de la ciudad de Cartagena. Revista Ciencias Biomédicas, 9(2), 120 130.

Recibido: 2 de mayo de 2020 Aprobado: 21 de junio de 2020

Autor de correspondencia: Elín Yohana Manrique-Julio elinmanriqueuis@hotmail.com

Editor: Inés Benedetti. Universidad de Cartagena-Colombia.

Copyright: (C) 2020. Manrique, E., Lozada, I., García, M. Este es un artículo de acceso abierto, distribuido bajo los términos de la licencia https://creativecommons.org/licenses/by-nc$\underline{\mathrm{sa} / 4.0 / \text { la cual permite el uso sin restricciones, }}$ distribución y reproducción en cualquier medio, siempre y cuando el original, el autor y la fuente sean acreditados.

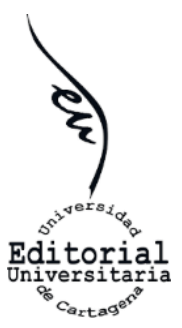

\section{Frecuencia de patologías orgánicas derivadas del consumo de sustancias psicoactivas en pacientes de una Unidad de Cuidados Intensivos de la ciudad de Cartagena}

\author{
Frequency of organic pathologies derived from the consumption \\ of psychoactive substances in patients of an Intensive Care Unit \\ in the city of Cartagena.
}
Elín Yohana Manrique-Julio ${ }^{1}$ Iván David Lozada-Martinez ${ }^{2}$, María Cecilia García- Espiñeira $^{3}$ (D)

\author{
${ }^{1}$ Fatoxacol, Barranquilla, Colombia. \\ ${ }^{2}$ Pregrado, Facultad de Medicina, Grupo Centro de Investigaciones Médico-Quirúrgicas, Universidad de \\ Cartagena, Colombia. \\ ${ }^{3}$ Departamento de Ciencias Básicas, Maestría en Toxicología, Facultad de Medicina, Universidad de \\ Cartagena, Colombia.
}

\section{RESUMEN}

Introducción: el consumo de sustancias psicoactivas se muestra como una de las principales causas de sobrecosto en unidades de cuidados intensivos en el mundo. Desde el ámbito toxicológico, la decisión de un tratamiento depende de la severidad de la intoxicación específica, por lo tanto, evaluar la dinámica poblacional a nivel local respecto a este tema, facilitará el abordaje de este tipo de pacientes con disminución de complicaciones, disminución de costos, y seguimiento en materia de la eficacia de políticas públicas contra la lucha de las drogas.

Objetivo: determinar la frecuencia de patologías orgánicas derivadas del consumo de sustancias psicoactivas en pacientes hospitalizados en una unidad de cuidados intensivos de la ciudad de Cartagena.

Métodos: se realizó un estudio observacional de corte transversal, en población adulta. Se revisó la historia clínica de todos los pacientes que ingresaron a la institución ajustados a los criterios de inclusión y exclusión. Se aplicó como herramienta el cuestionario del Sistema Interamericano de Datos Uniformes Sobre Consumo de Drogas. Se realizó test de sustancias psicoactivas. Los datos se analizaron en términos de frecuencia para establecer información relevante.

Resultados: se obtuvieron 80 casos de los cuales $75 \%$ eran hombres y $25 \%$ mujeres, el consumo de sustancias psicoactivas fue mayor en hombres. La mayoría de los casos estudiados procedían de la ciudad de Cartagena (70 \%) y vivían en unión libre $(54 \%)$. Las sustancias psicoactivas más consumidas fueron la cocaína (67\%) y la marihuana $(15 \%)$, y las patologías más comunes asociadas al consumo fueron cardiovasculares, cerebrovasculares y enfermedades infecciosas.

Conclusiones: las patologías de tipo cardiovascular, cerebrovascular y las enfermedades infectocontagiosas son las que afectan con mayor frecuencia a la población consumidora de sustancias psicoactivas ingresada a unidades de cuidados intensivos, siendo el consumo de cocaína más frecuente en la población masculina. 
Palabras Clave: toxicología; Unidades de Cuidados Intensivos; detección de abuso de sustancias; drogas ilícitas; enfermedad.

\section{ABSTRACT}

Introduction: the consumption of psychoactive substances is shown as one of the main causes of cost overruns in intensive care units in the world. From the toxicological field, the decision of a treatment depends on the severity of the specific poisoning, therefore, evaluating the population dynamics at the local level regarding this issue, will facilitate the approach of this type of patients, reduction of complications, costs, and monitoring of the effectiveness of public policies of the fight against drugs.

Objective: to determine the frequency of organic pathologies derived from the consumption of psychoactive substances in patients admitted to an intensive care unit of the city of Cartagena.

Methods: an observational cross-sectional study was carried out in an adult population. The medical records of all patients admitted to the institution adjusted to the inclusion and exclusion criteria were reviewed. The questionnaire of the Inter-American System of Uniform Data on Drug Use was applied as a tool. Psychoactive substances test was performed. The data was analyzed in terms of frequency to establish relevant information.

Results: 80 cases were obtained, of which $75 \%$ were men and $25 \%$ women, the consumption of psychoactive substances was higher in men. Most of the cases studied came from the city of Cartagena (70 \%) and lived-in common law $(54 \%)$. The most widely used psychoactive substances were cocaine (67 $\%$ ) and marijuana (15\%), and the most common pathologies associated with consumption were cardiovascular, cerebrovascular, and infectious diseases.

Conclusion: cardiovascular, neurologic and infectious diseases are the ones that most frequently affect the population that uses psychoactive substances admitted to intensive care units, with cocaine consumption being more frequent in male population.

Keywords: toxicology; Intensive Care Units; substance abuse detection; illicit drugs; disease. 


\section{INTRODUCCIÓN}

En Colombia la lucha contra las drogas plantea el reto más grande al que se ha enfrentado el país debido a la violencia derivada del mismo (1). El Observatorio Nacional de Drogas lidera la generación de conocimiento sobre el uso de drogas, a través de la realización de estudios en la población general, escolar y universitaria, con base en metodologías validadas internacionalmente que permiten la comparación con países de la región, con el propósito de contar con un panorama aproximado del problema de consumo de drogas en el país (2). En el año 2013, el Estudio Nacional de Consumo de Sustancias Psicoactivas realizado a una muestra de 32605 personas, concluyó que "existe un aumento del consumo global de drogas ilícitas (marihuana, cocaína, bazuco, éxtasis o heroína) en comparación con el estudio realizado en el 2008, pasando de una prevalencia de $8,8 \%$ en el 2008 , a $12,2 \%$ en el 2013". La marihuana sigue siendo la droga ilícita de mayor consumo en el país con una prevalencia que pasó de 2,1\% en el 2008, a un 3,3\% en el 2013, y, de hecho, el incremento en el grupo de drogas ilícitas se explica por el aumento en el uso de marihuana $(3,4)$.

Por otra parte, el Informe Mundial sobre Drogas del año 2018 señala un aumento en el número de consumidores de drogas, haciendo énfasis en el impacto sobre la salud en términos de sus consecuencias devastadoras, determinando la magnitud del daño provocado por el consumo de drogas en términos de años de vida ajustados en función de la discapacidad (4-6). De acuerdo con lo anterior, se estimó que en el año 2015 se perdieron 28 millones de años de vida "sana" en todo el mundo como resultado de la muerte prematura y la discapacidad causadas por el uso de drogas (5).

Desde el ámbito local, la ciudad de Cartagena de Indias, Colombia, en su plan de salud mental 20162019 articula dentro de sus objetivos específicos la conformación de redes de apoyo social e institucional, con el fin de implementar acciones intersectoriales para la promoción de factores protectores en salud mental, y para la prevención de factores de riesgo en violencia y en consumo de sustancias psicoactivas (6). No obstante, los estudios que miden la frecuencia de enfermedades relacionadas al consumo de sustancias psicoactivas y aspectos sociodemográficos de la ciudad y la región son limitados.

El consumo de sustancias psicoactivas también se muestra como una de las principales causas de sobrecosto en unidades de cuidados intensivos en el mundo, tal y como lo demostraron Westerhausen et al (7), donde 611 admisiones relacionadas a estos eventos, generaron costos por más de 74 millones de dólares (7). Lo previo, acompañado de la carga de enfermedad resultado de las complicaciones del consumo, culmina en la disminución de la capacidad funcional de los afectados. Las enfermedades cardiovasculares, neurológicas e infecciosas, constituyen las principales complicaciones del consumo de sustancias psicoactivas, mismas, que per se tienen una enorme carga de enfermedad (8). Desde el ámbito toxicológico, la decisión de un tratamiento dependerá de la severidad de la intoxicación específica, por lo tanto, evaluar la dinámica poblacional a nivel local respecto a este tema, facilitará el abordaje de este tipo de pacientes (principalmente en los departamentos de urgencias y cuidado crítico), disminución de complicaciones, disminución de costos, y seguimiento en materia de la eficacia de políticas públicas contra la lucha de las drogas (9).

La descripción de la distribución, frecuencia y tendencias de la enfermedad en las poblaciones, la identificación de la magnitud y dinámica de las necesidades de salud, y la identificación de la magnitud, vulnerabilidad y formas de control de los problemas de salud, hacen parte de los principales usos de la epidemiologia. Lo anterior, permite recopilar información sobre posibles factores de riesgo o factores protectores que impactan sobre la historia natural de alguna enfermedad, o también pueden ayudar a evaluar la eficacia y seguridad de una intervención terapéutica (10). En este orden de ideas, el objetivo de este estudio fue establecer a nivel local, la prevalencia de patologías derivadas del consumo de sustancias psicoactivas, en pacientes 
hospitalizados en una unidad de cuidados intensivos, y su relación en el desarrollo de patologías orgánicas.

\section{MÉTODOS}

Se llevó a cabo un estudio observacional de corte transversal, en una unidad de cuidados intensivos de Cartagena de Indias, Colombia; entre el 1 de enero y el 31 de diciembre de 2017. Se investigaron las características sociodemográficas de los pacientes con test positivo para sustancias psicoactivas que ingresaron a la institución, para determinar cuáles sustancias psicoactivas se encontraron con mayor frecuencia y su relación en el desarrollo de patologías orgánicas, y establecer la prevalencia de patologías orgánicas relacionadas con el uso de sustancias psicoactivas en los pacientes estudiados. La población de estudio estuvo conformada por todos los pacientes adultos entre 18 y 64 años que ingresaron a la unidad, que tenían sospecha clínica o antecedentes de consumo de sustancias de abuso y que presentaron patologías de tipo orgánico asociadas. Se excluyeron menores de edad y pacientes mayores de 65 años, obteniéndose 80 pacientes en total.

El estudio fue avalado por el comité de ética de la institución, respetó la Declaración de Helsinki, y se clasificó en la categoría de investigación sin riesgo, según el artículo 11 de la Resolución 8430 de 1993 del Ministerio de Salud. Todos los pacientes o sus familiares firmaron el consentimiento informado. El manejo de los datos obtenidos de las historias clínicas se realizó según lo dispuesto en la Ley 23 de 1981 del Congreso de Colombia y la Resolución 1995 de 1999 del Ministerio de Salud.

La unidad de análisis fue la historia clínica de todos los pacientes seleccionados. Se aplicó como herramienta de recolección de datos el cuestionario del Sistema Interamericano de Datos Uniformes Sobre Consumo de Drogas (SIDUC). Se realizó test de sustancias psicoactivas (One Step Multi-Drug, Multi-Line Screen Test Device), que permite la detección de 10 SPA en orina humana.

\section{Análisis estadístico}

La información se almacenó y analizó por medio del programa Microsoft Excel ${ }^{\circledR} \quad$ (Microsoft Corporation, Redmond, Washington, USA). Los datos se analizaron en términos de frecuencia para establecer información relevante. Los resultados obtenidos se compararon con los datos publicados por fuentes científicas como: SIVIGILA, Informe Mundial de Drogas 2017 y Estudio Nacional de Consumo de Sustancias Psicoactivas en Colombia 2013.

\section{RESULTADOS}

Todos los pacientes se encontraban afiliados al SGSS-S. El $65 \%(\mathrm{n}=52)$ pertenecían a Empresas Promotoras de Salud (EPS) subsidiadas y el $35 \%$ $(n=28)$ a EPS contributivas. El $70 \%(n=56)$ procedía de la ciudad de Cartagena y $30 \%(n=24)$ restante procedía de diferentes municipios del Departamento de Bolívar. El $75 \%(n=60)$ eran hombres y el $25 \%$ $(\mathrm{n}=20)$ mujeres. Con relación a la distribución por grupos etarios el $25 \%(\mathrm{n}=20)$ se encontraba comprendido entre los 40 a 49 años, el $23,75 \%$ $(\mathrm{n}=19)$ entre los 50 a 59 años y el $20 \%(\mathrm{n}=16)$ entre 30 a 39 años, (Figura 1).

En la relación de variables estado civil y género, el $54 \%(\mathrm{n}=43)$ de los pacientes convivían en unión libre, $23 \%(n=18)$ eran solteros, $19 \%(n=15)$ eran casados y solo el $4 \%(\mathrm{n}=3)$ eran individuos separados o divorciados. Los hombres tuvieron mayor tendencia a ser consumidores de sustancias psicoactivas que las mujeres sin importar el estado civil. En los solteros, no se encontró una diferencia significativa entre el de consumo de sustancias psicoactivas y el género. Con respecto a la situación ocupacional; los pacientes que mostraron un mayor consumo de sustancias $(47,5 \%, \mathrm{n}=38)$ trabajaban a tiempo parcial, seguido por un $31,2 \%(\mathrm{n}=25)$ que trabajaban de tiempo completo. En estudiantes, amas de casa o desempleados, los porcentajes fueron menores. 


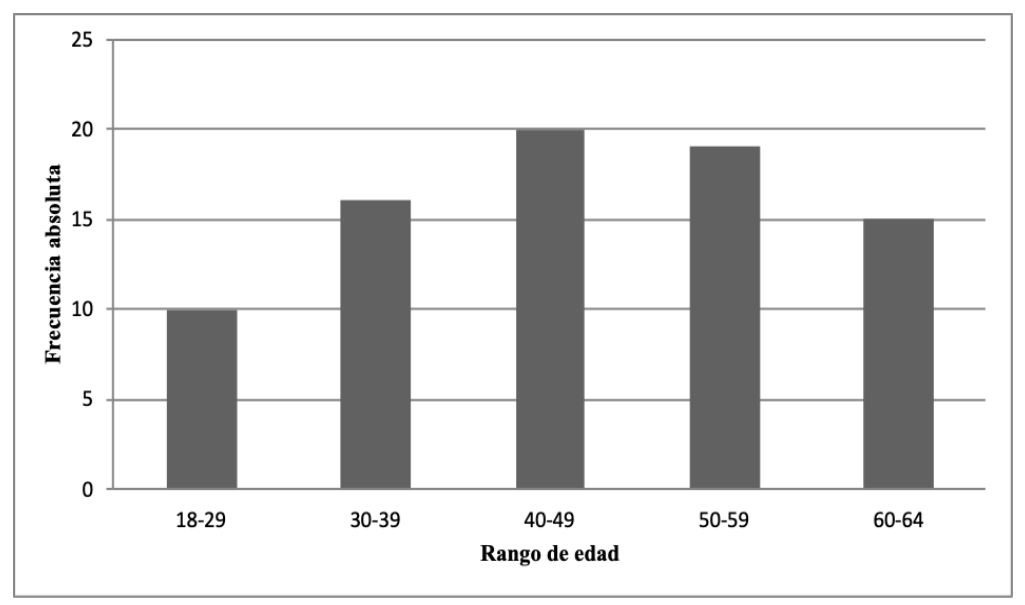

Figura 1. Frecuencia absoluta de pacientes con patologías orgánicas según rango de edad

La sustancia psicoactiva de consumo más frecuente por la población estudiada fue la cocaína, detectada en $67 \%(n=53)$ de los pacientes estudiados, seguido por la marihuana en $15 \%(n=12)$ y los derivados opioides en $11 \%(n=9)$. En menor porcentaje: benzodiacepinas $(5 \%, \quad n=4)$ barbitúricos y metanfetaminas, ambas con $1 \%(n=1)$, (Figura 2).

Al analizar qué patologías se presentaron en la población objeto de estudio, se encontró que el $20 \%$ $(\mathrm{n}=16)$ de los pacientes tuvieron diagnóstico definitivo de infarto agudo de miocardio y otro $20 \%$ $(n=16)$ de angina inestable, siendo estos los diagnósticos más frecuentes. Los anteriores fueron seguidos por: accidente cerebrovascular isquémico, neumonía adquirida en la comunidad y VIH/SIDA con representación diagnóstica en igual porcentaje: $8,75 \%,(n=7)$. Con menor porcentaje, se encontraron el asma, síncope, enfermedad pulmonar obstructiva crónica, hepatitis B y pancreatitis, entre otras (Figura $3)$.

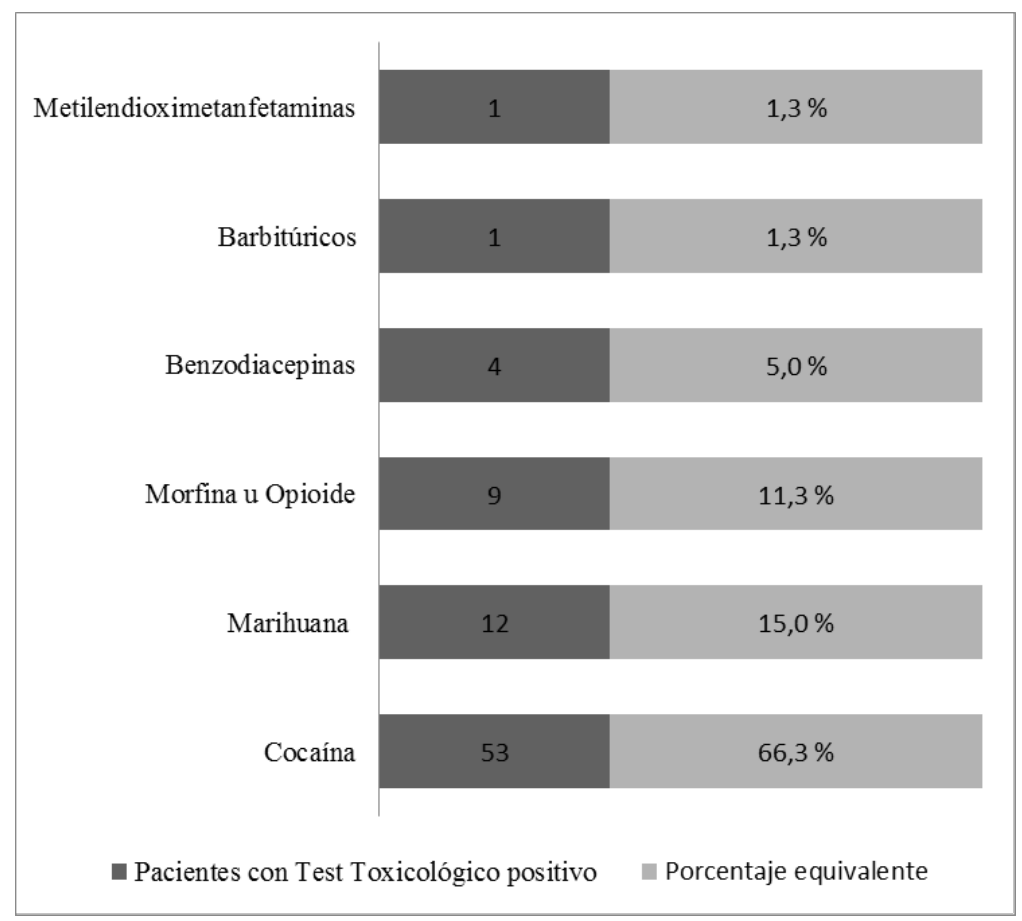

Figura 2. Distribución de consumo de sustancias psicoactivas según resultado de test toxicológico 


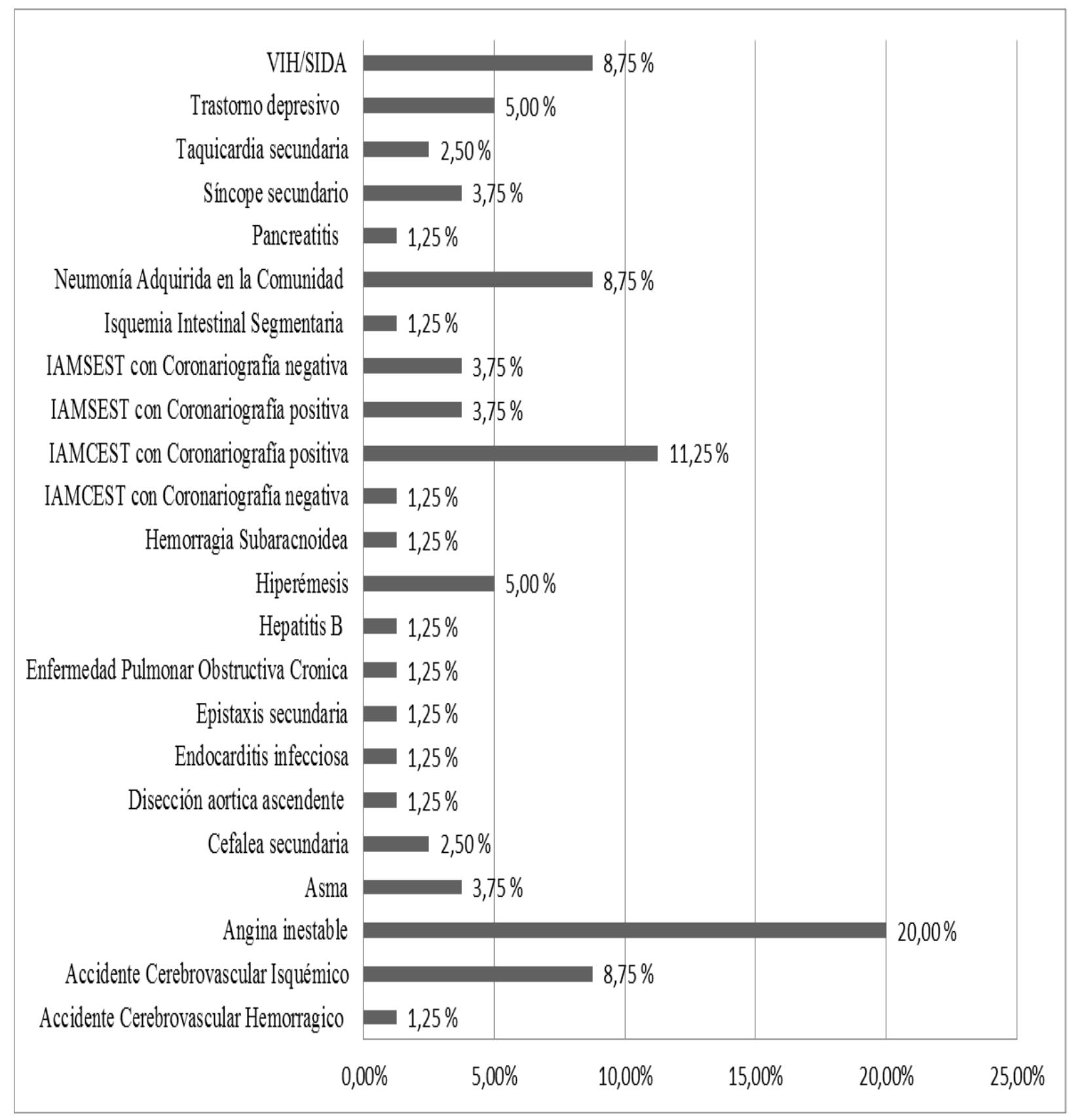

Figura 3. Distribución porcentual de patologías presentadas derivadas del consumo de sustancias psicoactivas. IAMSEST: Infarto Agudo de Miocardio Sin Elevación del Segmento ST- IAMCEST: Infarto Agudo de Miocardio Con Elevación del Segmento ST.

Finalmente, al relacionar las patologías diagnosticadas con la sustancia psicoactiva detectada, y teniendo presente que las más frecuentes fueron cocaína $(67 \%, \mathrm{n}=53)$, marihuana $(15 \%, \mathrm{n}=12)$ y opioides $(11 \%, \mathrm{n}=9)$, se observó que en el $67 \%(n=36)$ de los pacientes a los cuales se les detectó cocaína, las patologías predominantes corresponden a infarto agudo de miocardio y angina inestable las cuales se presentan en igual porcentaje: $30,19 \%, \quad(n=11)$, seguidos por el accidente cerebrovascular de tipo isquémico con 13,21\% $(n=5),($ Figura 4). 
Las patologías más frecuentes relacionadas al consumo de marihuana fueron: neumonía adquirida en la comunidad $(33,33 \%, \mathrm{n}=4)$, hiperémesis $(33,33$ $\%, \mathrm{n}=4)$, asma $(16,67 \%, \mathrm{n}=2)$, síncope y EPOC $(8,33 \%, \mathrm{n}=1)$. Mientras que las patologías relacionadas al uso opioides fueron: enfermedades infecciosas como VIH/SIDA 77,78 \% $(n=7)$, hepatitis $\mathrm{B}$ y endocarditis infecciosa, ambas con $11,11 \%(n=1)$.

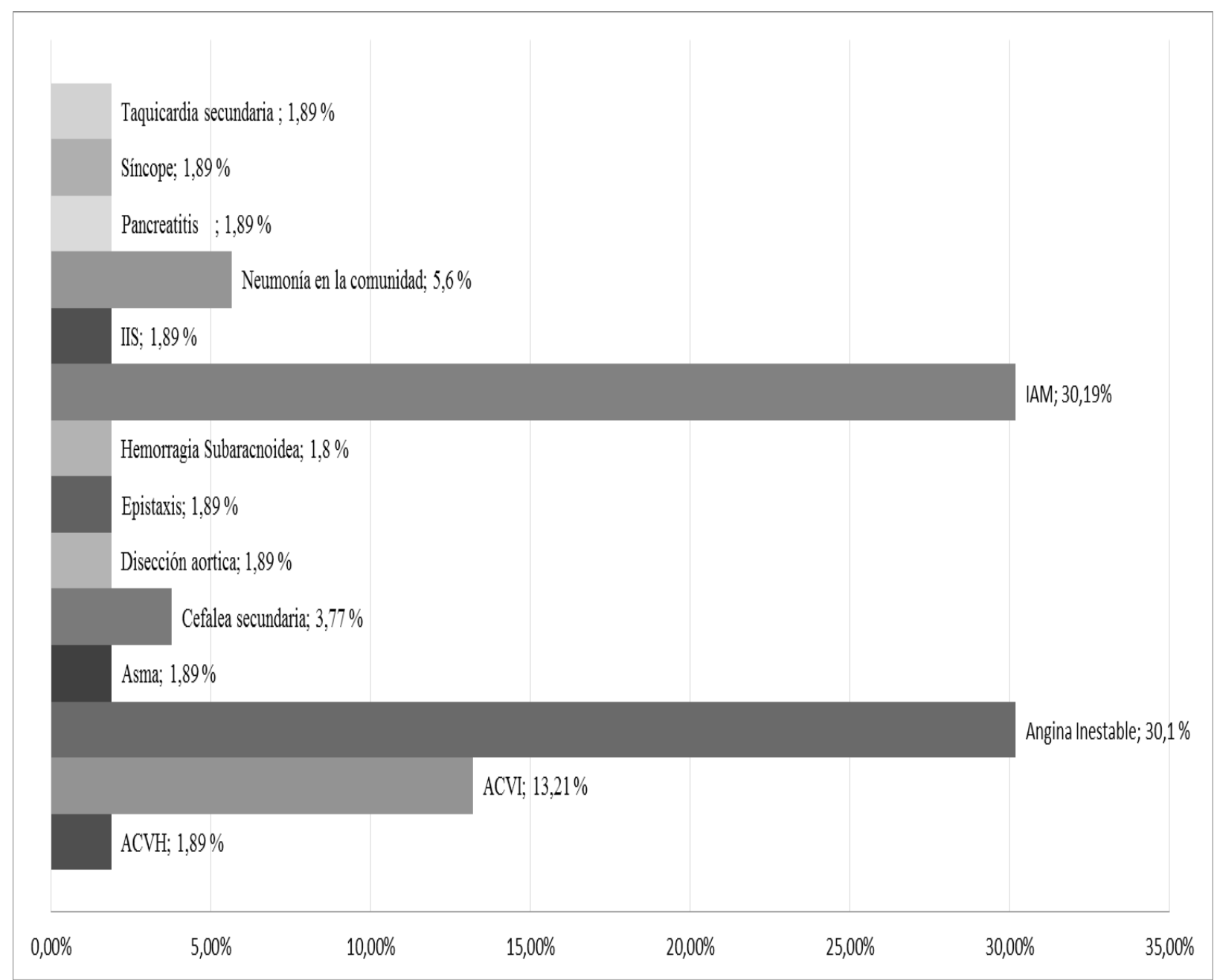

Figura 4. Relación porcentual de las patologías presentadas derivadas del consumo de cocaína. IIS: Isquemia Intestinal Segmentaria. IAM: Infarto Agudo de Miocardio. ACVI: Accidente Cerebrovascular Isquémico. ACVH: Accidente Cerebrovascular Hemorrágico.

\section{DISCUSIÓN}

En el Informe sobre el Consumo de Drogas en las Américas correspondiente al año 2019, se menciona que los patrones del consumo de Cannabis han estado evolucionando rápidamente en todo el hemisferio occidental (11). En la población general, el aumento fue evidente en al menos seis de los siete países que proporcionaron estos datos. Junto a tales aumentos, también se observa el consumo de marihuana a edades cada vez más tempranas, mientras que la percepción general del riesgo asociada al consumo está disminuyendo. Comportamiento similar es observado en el presente estudio, donde la marihuana resultó como la segunda sustancia de mayor consumo, siendo un antecedente común en algunos de los individuos que presentaron neumonía adquirida en la comunidad. Si bien la marihuana medicinal se comercializa cada 
vez más como segura y saludable, los riesgos y las consecuencias de su uso a largo plazo y otras formas de Cannabis podrían no estar claras para el público en general (11), tal y como lo evidenciaron Richards et al (2019), donde encontraron una fuerte asociación entre el consumo de Cannabis y un incremento en el riesgo de enfermedad cardiovascular aterosclerótica establecida, principalmente de síndrome coronario agudo (12).

En el mismo informe mencionado previamente, se estimó que la prevalencia más alta en la región de las Américas la lidera Estados Unidos, con un consumo del 1,9\% en la población general, seguido por Argentina y Uruguay $(1,6 \%)$ y Canadá $(1,5 \%)$. México, Costa Rica, Belice, Chile, Brasil, Colombia y Venezuela están en un rango intermedio de consumo de cocaína (más de $0,5 \%$ a $1,1 \%$ ) (11). Si bien la cocaína y sus derivados son algunas de las drogas más utilizadas en América del Sur, también son algunas de las sustancias más adulteradas. Los adulterantes son compuestos químicos que tienen alguna propiedad farmacológica similar a la droga de abuso y se agregan para potenciar su efecto. Comúnmente estos son más peligrosos que la propia droga de abuso. En el caso de la cocaína, los adulterantes más usados son la cafeína, la lidocaína $y$ el levamisol (11). Estas afirmaciones se correlacionan con los hallazgos del presente estudio, donde el consumo de cocaína prevaleció en los casos de trastornos cardiovasculares, resultado de la actividad intensa de catecolaminas por un estímulo exagerado al sistema nervioso, lo que conduce a una potente vasoconstricción, taquicardia y predisposición a arritmias fatales (13). En caso de pacientes con factores de riesgo cardiovasculares, este proceso precipita el inicio de angina inestable, infarto agudo de miocardio, disección aórtica, o miocarditis, entre otras (14).

A pesar de que solo se identificó que el $11.3 \%$ de los pacientes ingresados consumieron opioides, es relevante destacar que las patologías encontradas en estos casos son similares a las reportadas en la literatura, donde las infecciones bacterianas agudas, VIH/SIDA, y hepatitis, fueron las más frecuentes (14). Lo anterior, dependerá de la vía de administración, siendo las más comunes la ingestión, insuflación nasal, inhalación e inyección por vía intravenosa, intramuscular o subcutánea.

Particularmente desde el punto de vista sociodemográfico, los grupos etarios donde el consumo de drogas fue más frecuente fue el comprendido entre 40 y 49 años, seguido de los pacientes con edades entre 50 y 59 años, marcando una clara diferencia con los resultados del Estudio Nacional de Consumo de sustancias psicoactivas del año 2013, que señala que el mayor consumo se presenta en el grupo de 18 a 24 años, con una tasa del $8,7 \%$, seguido por los adolescentes con una tasa de $4,8 \%$ y el rango de 25 a 34 años, con una prevalencia de $4,3 \%$ para el período estudiado (2). Estos datos pueden tener un valor potencialmente significativo, puesto que la prevalencia de consumo en grupos etarios mayores puede correlacionarse con el envejecimiento de drogadictos de larga data, lo que elevaría ampliamente el riesgo de presentar complicaciones orgánicas derivadas del consumo de sustancias psicoactivas, impactando directamente en costos de salud y tasas de morbimortalidad local (15).

Otro dato que es importante mencionar es el análisis de las sustancias consumidas, puesto que el incremento en la incidencia y prevalencia en grupos etarios mayores puede provenir de la adquisición de fármacos por receta médica, como los opioides y las benzodiacepinas en caso de manejo de patologías neurológicas o psiquiátricas, algo muy frecuente en este grupo poblacional (16).

El análisis de la variable género permite observar que la proporción de consumo de hombres frente al de las mujeres es similar a la reportada en la literatura científica, a pesar de existir estudios que afirman que el género femenino ha incrementado el consumo de sustancias psicoactivas, principalmente por el uso de tranquilizantes y somníferos $(17,18)$. Los resultados arrojados por este estudio pueden ser el reflejo implícito de la inequidad de oportunidades en el ámbito sociolaboral con respecto al género, debido a que la alta proporción de consumo por parte del género masculino puede derivarse de las 
condiciones estresantes frente a la fuerte carga laboral $(17,18)$. Teniendo en cuenta que en la región del Caribe colombiano esta inequidad es sumamente marcada, ya que generalmente el hombre es la base económica del hogar incluso en edades más avanzadas, lo que puede considerarse un factor de riesgo.

Lo anterior se encuentra íntimamente relacionado con el hecho de que aquellas personas que viven en unión libre tienden a ser los mayores consumidores (54\%). Se puede destacar que en el caso del género femenino en la misma región, el hecho de desempeñarse de forma forzada como ama de casa, producto de creencias conservadoras y déficit de políticas que aumenten la probabilidad de un vínculo laboral eficiente, otorga una responsabilidad pasiva pavorosa en el orden del hogar, lo que a su vez instaura un espacio biopsicosocial muy reducido, influyendo negativamente en la salud mental de las mismas y pudiendo explicar así el incremento de consumo de sustancias psicoactivas, específicamente de tranquilizantes y somníferos (19). Esto permite inferir que se necesita un cambio urgente $y$ sistémico en aquellas políticas que definen los criterios de acceso no solo al ámbito laboral, sino también en la parte social, cultural y de sanidad para romper la barrera de la inequidad, e igualar condiciones y responsabilidades entre los dos géneros que ocasionen mejoras en la esfera psicosocial de la población en general, pudiendo impactar enormemente en las tasas de consumo de sustancias psicoactivas (20).

De forma interesante, se encontró una diferencia significativa de la prevalencia sobre el consumo departamental de sustancias psicoactivas, donde la marihuana era la sustancia más consumida. En el presente estudio, la cocaína fue detectada en el $67 \%$ de los pacientes seguida por la marihuana en un 15 $\%$, y un $11 \%$ representado por los derivados opioides (21). Esto podría indicar claramente dos aspectos, primero, la producción y distribución de la cocaína se ha incrementado considerablemente en el departamento, y segundo, se ha facilitado el acceso a esta droga probablemente por ollas de microtráfico o nuevos procesos de distribución.

En este orden de ideas, podemos afirmar que el consumo de drogas es un problema de salud pública en Bolívar. Es necesaria la creación de protocolos de seguimiento por parte del ámbito institucional y local, con el fin de realizar un diagnóstico oportuno en el paciente drogadicto, a fin de establecer programas eficaces a través de su entidad promotora de salud. Se pudo observar que el hecho de que estos individuos sean consumidores de larga data evidencia la falta de eficacia de programas de rehabilitación de drogadicción. Además, se pudo demostrar que la prueba de identificación de sustancias psicoactivas es un método sencillo y rápido de diagnóstico que permite enfocar de forma precoz la relación sustancia-complicación, para predecir el riesgo de morbimortalidad.

Como recomendaciones, se sugiere indicar a las instituciones prestadoras de salud la importancia de socializar las fichas de vigilancia epidemiológicas y su reporte efectivo para generar estadísticas institucionales y locales que contribuyan con la generación de políticas públicas eficaces en salud. Este tipo de estudios motiva el cambio a nivel de la legislación gubernamental hacia la identificación temprana del problema de la drogadicción y los factores que influyen en estas. Los factores económico, social, cultural, educativo y sanitario son variables que siempre se deben tener en cuenta en la consulta para calcular la probabilidad de consumo, principalmente en grupos de riesgo. Desde la academia, motivar la conformación del observatorio de drogas a nivel regional que permita tener la disposición de datos fiables para evaluar la situación del consumo de drogas, puesto que no se debe olvidar el impacto que tiene esta situación en el campo de la salud pública. Este estudio tuvo algunas limitaciones, como ser unicéntrico y tomar muestra solamente de personas en una unidad de cuidados intensivos. Se necesitan estudios multicéntricos con muestras mayores, para apoyar la evidencia epidemiológica. 
En conclusión, las patologías cardiovasculares, neurológicas y las enfermedades infectocontagiosas son las que afectan con mayor frecuencia a los pacientes adultos con sospecha clínica $o$ antecedentes de consumo de sustancias psicoactivas y que presentaron patologías orgánicas asociadas, en una unidad de cuidados intensivos de Cartagena de Indias; prevaleciendo en la población masculina el consumo de cocaína.

CONFLICTOS DE INTERESES: Los autores declaran no tener conflictos de interés.

AGRADECIMIENTOS: A la Clínica ESTRIOS, de la ciudad de Cartagena. Colombia, por su apoyo al facilitar la obtención de la información necesaria para realizar este estudio.

\section{REFERENCIAS}

1. Scoppetta O. Consumo de drogas en Colombia: características y tendencias. Colombia: Editorial Guadalupe S.A.; 2010. pags. 1-58.

2. Observatorio de Drogas de Colombia. Estudio nacional de consumo de sustancias psicoactivas en Colombia 2013. Colombia: ODC. 2014.

3. Dirección Nacional de Estupefacientes. Estudio nacional de consumo de sustancias psicoactivas en Colombia 2008. Colombia: DNE. 2009.

4. Oficina de las Naciones Unidas contra la Droga y el Delito. Informe mundial sobre las drogas 2016. UNODC. 2016.

5. Oficina de las Naciones Unidas contra la Droga y el Delito. Informe mundial de drogas 2018: crisis de opioides, abuso de medicamentos y niveles récord de opio y cocaína. Estados Unidos: UNODC. 2018.

6. Departamento Administrativo Distrital de Salud. Programa de Salud Mental de Cartagena. Colombia: DADIS. 2016.

7. Westerhausen D, Perkins AJ, Conley J, Khan BA, Farber M. Burden of Substance Abuse-Related Admissions to the Medical ICU. Chest. 2020; 157(1):61-66.

8. Organización Mundial de la Salud. Carga mundial de morbilidad [Internet]. Disponible en: https://www.who.int/topics/global_burden_of_disease/e s/

9. Reisinger A, Rabensteiner J, Hackl G. Diagnosis of acute intoxications in critically ill patients: focus on biomarkers - part 1: epidemiology, methodology and general overview. Biomarkers. 2020; 25(1):9-19.

10. Hernández-Ávila M, Garrido-Latorre F, López-Moreno S. Diseño de estudios epidemiológicos. Salud Pública de México. 2000; 42(2):144-154.

11. Organización de Estados Americanos. Informe sobre el consumo de drogas en las Américas, 2019 [Internet]. Disponible en: http://www.cicad.oas.org/main/pubs/Informe $\% 20$ sobre $\% 20 \mathrm{el} \% 20$ consumo $\% 20 \mathrm{de} \% 20$ drogas $\% 20$ en $\% 20$ las $\% 2$ 0Am\%C3\%A9ricas\%202019.pdf

12. Richards JR, Bing ML, Moulin AK, Elder JW, Rominski RT, Summers PJ, et al. Cannabis use and acute coronary syndrome. Clin Toxicol (Phila). 2019; 57(10):831-841.

13. Schwartz BG, Rezkalla S, Kloner RA. Cardiovascular effects of cocaine. Circulation 2010; 122(24):2558-69.

14. Donroe JH, Tetrault JM. Substance Use, Intoxication, and Withdrawal in the Critical Care Setting. Crit Care Clin. 2017; 33(3):543-558.

15. Observatorio Europeo de las Drogas y las Toxicomanías. Drogas en el punto de mira. EMCDDA. 2008.

16. Sarkar S, Parmar A, Chatterjee B. Substance use disorders in the elderly: A review. J Geriatr Ment Heal. 2015; 2(2):74-82.

17. Sánchez L. Prevención del consumo de drogas con perspectiva de género: recomendaciones con base a la evidencia. Alicante: Quinta impresión; 2014. pags. 1-99.

18. Junta Internacional de Fiscalización de Estupefacientes. Informe de la Junta Internacional de Fiscalización de Estupefacientes correspondiente a 2018. Viena: JIFE. 2018.

19. Sánchez R, Fernández M. El perfil de los consumidores de drogas. Ciencia. 2014; 65(1): 18-25.

20. Ministerio de Salud y Protección Social. Plan nacional para la atención del consumo de sustancias con enfoque de salud pública, 2014-2021. Colombia: MSPS. 2014. 
21. Observatorio de Drogas de Colombia. Atlas de la caracterización regional de la problemática asociada a las drogas ilícitas en el departamento de Bolívar. Colombia: ODC. 2016. 